MUZIKOLOSKI ZBORNIK - MUSICOLOGICAL ANNUAL XVII/1, LJUBLJANA 1981

UDK 78.071.22(443.611)"1830"

\title{
LES CONCERTS A PARIS EN 1830
}

François Les u re (Paris)

La reconstitution de la vie des concerts au début du XIX ${ }^{\mathrm{e}}$ siècle présente de nombreuses difficultés dès que l'on veut quitter les généralités et l'envisager sur un plan quantitatif. Les programmes ne sont qu'exceptionnellement conservés et la presse ne recouvre que très partiellement la réalité. A l'occasion d'une enquête collective sur la musique à Paris en 1830 et 1831, j'ai tenté de faire un bilan de deux années de concerts dans cette ville d'après toutes les sources existantes et de dégager ainsi pour une période précise la sociologie de l'institution. Ce dépouillement, qui a porté sur une vingtaine de journaux, a fait apparaître un total (évidemment très incomplet) de 129 concerts pour 1830 et 142 pour 1831. Dans le déchiffrage de cette statistique, il faut tenir compte des conséquences de la révolution de juillet pour la première année et de l'épidémie de choléra pour la seconde. Mais la répartition de ces concerts durant l'année montre suffisamment que la saison culmine entre décembre et mai avec un maximum en mars et avril. Il n'y a cependant pas d'arrêt total de l'activité durant l'été.

Aucune coordination n'apparaît dans leur échelonnement: jusqu'à cinq concerts dans la même journée alors qu'aucun n'est organisé à de nombreuses dates. Le jour privilégié est le dimanche, seul jour de relâche des deux théâtres lyriques principaux. C'est celui que choisit toujours la Société des concerts du Conservatoire, alors que l'Institution de Choron a l'habitude du jeudi. Ces deux séries ont lieu non pas le soir mais à deux heures de l'après-midi. Tandis que les jours de semaine les concerts débutent entre sept et huit heures trente, le dimanche permet deux tranches horaires: la première à une heure trente ou deux heures, la seconde à huit heures ou huit heures trente.

Les prix d'entrée sont assez variables, selon le caractère $\mathrm{du}$, concert, la salle et la qualité des interprètes: de $2 \mathrm{f}$. 50 à $12 \mathrm{f}$. Il est fréquent qu'il y ait deux prix: 5 et $10 \mathrm{f}$. Le record appartient au pianiste Hummel: 8, 10 et 12 f. Dans l'ensemble ces prix sont analogues à ceux que pratiquent les grands théâtres lyriques. Une bonne 
dizaine de salles accueillent la majorité des concerts. Leur contenance est généralement modeste, sauf la salle des Menus Plaisirs (un millier de places), réservée pratiquement à la Société des concerts du. Conservatoire, et la salle St-Jean à l'Hôtel de ville qu'utilisent certaines associations comme le Gymnase et l'Athénée musical. Beaucoup de concerts ont lieu dans les salons de grands facteurs de pianos: Dietz, Erard, Pape, Petzold, Pleyel. Les autres se partagent la salle Chantereine, peu confortable et de mauvaise acoustique, la salle Taitbout et parfois, la salle Cléry et celle du Wauxhall. Il faut mettre à part l'Institution de Choron, qui présente ses séances dans son local de la rue de Vaugirard, et le quatuor de Baillot, qui reste fidèle à l'Hôtel Fesch, rue St-Lazare. Enfin, certains concerts avaient lieu dans des théâtres: souvent «à bénéfice» (voir plus loin), notamment à l'Opéra comique et au Théâtre italien. L'événement le plus spectaculaire des années 30 et 31 fut l'apparition de Paganini, auquel fut exceptionnelle-ment offert l'Opéra et que nous envisagerons ci-dessous.

La cadre étant ainsi tracé, nous pouvons tenter d'établir des catégories, de dégager certains types différents de concerts. L'Institution royale de musique religieuse fondée par Choron et qui se posait en rivale $d u$ Conservatoire donnait depuis quatre ans des concerts publics consacrés exclusivement aux »chefs d'oeuvre des plus grands maîtres de toutes les écoles et de tous les temps". Pendant la saison 1829-30 elle ne donna pas moins de douze séances représentant un effort considérable puisqu'on y entendit: Samson, Judas Macchabée et d'importants extraits d'Alexander's Feast de Haendel, Die Israeliten in der Wüste de C. Ph. E. Bach, des oeuvres de Palestrina, Janequin, Mozart, Allegri, Marcello, les Sept paroles du Christ de J. Haydn ainsi que des extraits de Der Tod Jesu de C. H. Graun -répertoire qui se voulait en réaction contre le "genre vulgaire moderne» pratiqué au Conservatoire. La révolution de juillet, en supprimant une grande partie de sa subvention, devait être fatale à Choron. Celui-ci changea son nom pour tenter de se mettre au goût du jour et devint «Institution royale de musique classique, ancienne et moderne, sacrée et profane», mais ne put rétablir ses brillantes activités.

La Société des concerts du Conservatiore était alors de création récente (février 1828), mais son essor avait été rapide: en 1830 elle donna dix concerts sous la direction de Habeneck. Comme son histoire est assez bien connue, nous n'y insisterons pas, rappelant que la base de son répertoire comprenait les symphonies de Beethoven: les premières auditions de la quatrième et de la neuvième eurent lieu respectivement les 21 février 1830 et 27 mars 1831. Mais on y entendait aussi bien divers extraits d'Euryanthe de Weber, un concerto de piano de Kalkbrenner, des oeuvres religieuses de Cherubini, un solo de violoncelle de Romberg, des extraits d'opéras (Gluck, Rossini). Un concert entier fut consacré à Méhul et l'on ne rappellera que pour mémoire 
le fameux concert de Berlioz donné au profit des blessés de la révolution, le 5 décembre 1830, où fut exécutée la Symphonie fantastique.

D'autres associations avaient moins de prestige mais représentent mieux les possibilités offertes aux compositeurs et interprètes pour se faire connaître. L'Athénée musical fonctionnait ainsi à l'Hôtel de ville depuis décembre 1829 et se proposait à la fois de faire connaître des jeunes auteurs et d'offrir des oeuvres de grands maîtres. $\mathrm{H}$. Chélard $\mathrm{y}$ jouait le rôle principal et organisait chaque mois un concert. Voici, à titre d'exemple, deux de ses programmes:

Concert du 28 janvier 1830. Orchestre sous la direction de Bloc.

Scène composée par Elwart sur le motif de l'Exilé, chantée par Cambon. Symphonie concertante de Tulou pour flûte, hautbois, cor et basson.

Fantasie pour piano par Payer.

Variations pour le basson-alto, exécutées par Rikmans père, accompagné par Rikman fils, âgé de huit ans, au piano.

Romances de Panseron chantées par Cambon.

Air italien d'Amédée Ráoul, chanté par Mlle Beck.

Ouverture à grand orchestre de Rigel.

Fantasie pour harpe de Bochsa, exécutée par Mme Berbiguier, arrangée par lui pour symphonie à grand orchestre.

Air de Rosine du Barbier de Séville de Rossini, chanté par Mlle Olivier.

Air des Noces de Figaro de Mozart, chanté par Hurteaux.

Armide de Lully, scène de la Haine, et «Plus j’observe ces lieux», chantés par Mme Kretschmar.

Armide de Gluck, scène des Enfers, chantée par Mlle Bolard.

Armide de Rossini, air chanté par Mlle Kunze.

Concert du 18 février 1830. Orchestre dirigé par Bloc.

Ouverture à grand orchestre de Lefebvre.

Ouverture de Varney.

Fantaisie pour cor et piano de Ermel.

Air de basse de Héquet, chanté par Dalpi.

Air de basse composé et chanté par Vachon.

Plusieurs romances et chansonnettes.

Air de Joseph de Méhul. chanté par Cambon.

Deux mélodies de Berlioz sur des paroles de Th. Moore (Irish melodies): Hymne à l'éternel.

Ouverture à grand orchestre de Désormery.

Air de La Gazza ladra de Rossini, chanté par Louise Lebrun.

Duo de Tancredi de Rossini, chanté par Mlle Kunze et Boulanger.

Air de Tancredi chanté par Mlle Kunze.

Concerto de violon, exécuté par Schmitt, premier violon du grand duc de Hesse-Darmstadt.

Mme Pontaillié joue du piano.

Trio du Matrimonio secreto de Cimarosa chanté par Mme Lebrun et ses deux filles.

Chant des chasseurs de Robin des bois de Weber.

On trouve là les traits caractéristiques de ce type de concert: éclectisme, longueur et dissémination du répertoire, mélange de vocal et instrumental, goût pour les enfants prodiges et pour les extraits d'oeuvres lyriques. Une autre association -le Gymnase musical- sise également à l'Hôtel de ville et de création récente, avait à peu près 
les mêmes tendances, avec une préférence pour «toute musique sévère et classique». Le chef d'orchestre en était Tilmant. Ses concerts semblent avoir été moins nombreux et réguliers que ceux d'une association plus ancienne, la Société académique des enfants d'Apollon, dont la musique n'était pas l'unique préoccupation mais qui avait le privilège d'utiliser la salle des Menus plaisirs. On aura souligné le profil de ses concerts en reproduisant le programme de sa séance annuelle du, 20 mai 1830:

Première partie.

Ouverture de Rigel.

Impromptu analyse, trio bouffe des Précieuses ridicules de $\mathrm{H}$. Blanchard.

Solo de cor de X., exécuté par Rousselot.

Canon à trois voix de Garcia, chanté par Levasseur, Mmes Gebauer et Cinti-Damoreau.

»J'entends le signal du combat«, chant de guere de Panseron, chanté par Levasseur.

Trio pour piano, clarinette et basson de Brod.

Air des Voitures versées de Boieldieu chanté par Mlle Moncel.

Deuxième partie.

Concerto pour violon de Viotti, exécuté par Tilmant.

Scène et air de Rossini, chanté par Mme Cinti-Damoreau.

Solo d'alto et de viole d'amour, composé et exécuté par Urhan.

Quatuor de Rossini, chanté par Mme Cinti-Damoreau, Levasseur, Domange et Panseron.

Orchestre dirigé par Launer.

Quittons les associations pour aborder une modeste mais importante catégorie: celle de la musique de chambre. Outre Anton et Max Bohrer, dits les frères Bohrer, qui, dans leurs soirées musicales, programmaient notamment les derniers quatuors de Beethoven, le grand défenseur de ce genre alors ésotérique était le violoniste Pierre Baillot. Depuis quinze ans, il présentait trios, quatuors et quintettes de Boccherini, Haydn, Mozart, Beethoven, Onslow devant un public particulièrement attentif et motivé.

Enfin le type le plus fréquent de concert était celui qui était donné par des virtuoses: beaucoup de vedettes, tels F. Hiller, Moschelès, H. Herz, Pixis, A. Sowinski, Panseron, Mme Farrenc, Mazas, et aussi de moins connus, tels que Pechatschek, premier violon de la chapelle du grand-duc de Bade, et... jusqu'à G. Filippa, «élève de Paganini», violoniste âgé de quatorze ans. Là encore, quelques exemples sont nécessaires pour montrer combien leurs programmes étaient différents de ce qu'allait être vers, la fin du siècle un «récital».

Soirée musicale de Charles Schunke, 29 avril 1830.

Les Adieux, fantaisie pour harpe, composée et exécutée par Léon Gatayes. Duo de piano et violon, composé et exécutée par Habeneck aîné et Schunke. Romances composées et chantées par Panseron, accompagnées au hautbois par Brod.

Air d'Il Barbiere de Rossini, chanté par Mme Danvers.

Pot-pourri sur des thèmes d'Auber, exécuté par Schunke. 
$1^{\text {re }}$ partie du grand septuor pour piano, flûte, hautbois, cor, violoncelle, alto et contrebasse de Hummel.

Duo de Mathilde de Sabran de Rossini, chanté par Mme Danvers et Pellegrini. 3 e partie du septuor de Hummel.

Romances chantées par Domange.

Elégie pour alto avec accomp. de harpe de Mazas.

Ouverture des Mystères d'Isis de Mozart, arrangée pour 3 pianos à 12 mains, par Payer.

Concert donné par Ferdinand Hiller, 4 décembre 1831.

Symphonie à grand orchestre de F. Hiller.

Variations pour violon de Mayseder, exécutées par Urhan.

Duo de Semiramide ("Bella imago«) chanté par Stephen et Mme Raimbaux.

Concerto pour piano composé et exécuté par Hiller.

Cavatine du Barbier de Séville par Mme Raimbaux.

Solo de violoncelle par Franchomme.

Ouverture à grand orchestre pour Faust de Goethe de F. Hiller.

Romances inédites chantées par Mme Raimbaux.

Grand concerto à deux pianos de Kalkbrenner exécuté par l'auteur et F. Hiller.

Il faut faire une place spéciale aux onze concerts donnés, deux fois par semaine, par Paganini en mars et avril 1831. Fait exceptionnel, le tout nouveau directeur de l'Opéra avait eu le sens commercial de lui ouvrir son théâtre. Il y avait même effectué des travaux spéciaux pour augmenter le nombre des places (environ 1800) et prévu une mise en scène pour accroître l'effet théâtral de l'apparition du virtuose. On donnera ici le programme du concert du 20 mars 1831, qui fit plus de 20.000 f. de recettes:

Ouverture de Robin des bois.

Air chanté par Mllle Dorus (Rossini).

Concerto à trois temps (avec accomp. de triangle) composé exprès pour Paris par Paganini.

Fragment d'une symphonie de Hayden.

Trio de l'Inganno felice de Rossini, chanté par Levasseur, Alexis Dupont et Mille Dorus.

Recitativo et variazioni sequante tre arie, composés et exécutés par $\mathrm{Pa}-$ ganini sur une seule corde (la 4e).

Trio de l'Auberge de Bagnères de Catel.

Larghetto e variazioni sul rondo del Cenerentola, composés et exécutés par Paganini.

Laissant de côté les exercices publics d'élèves, présentés par certains professeurs (F. Stoepel, Egasse, Massimino), nous passerons enfin en revue les concerts de bienfaisance et concerts "à bénéfice», assez nombreux pour constituer une catégorie à part: "au bénéfice d'une famille pauvre», ou "d'une famille espagnole», "de la salle de l'asile St-Martin», «des jeunes orphelins», «des veuves, orphelins et blessés belges» (au lendemain de la révolution), «de plusieurs polonais», "d'un homme de lettres malheureux» et surtout quantité de concerts au bénéfice des victimes de juillet. Il y en eut même un qui fut donné par 24 musiciens placés dans le ventre d'une baleine! 
Quant aux concerts dits «à bénéfice», le mécanisme en est difficile à démonter. On a quelque peine à savoir qui prend l'initiative de telles séances, dont la recette va à des chanteurs ou des instrumentistes qui ne semblent pas spécialement en difficulté financière. Ainsi dans le cas de Mme Schroeder-Devrient, qui bénéficie, le 28 juin 1831, d'un tel concert, au cours duquel elle chanta elle-même. Tel n'était pas toujours le cas: les interprètes en étaient plutôt des collègues et des amis du bénéficiaire. Sous Charles $\mathrm{X}$, il arrivait que le roi ajoutât lui-même sur sa cassette une somme à la recette. C'est sans doute la seule forme de subvention directe du pouvoir à la vie du concert que l'on puisse relever à l'époque. Par ailleurs, loin de favoriser financièrement les concerts, les autorités les taxaient assez lourdement du fameux "droit des pauvres», dont des générations se sont plaint jusqu'aux approches du $\mathrm{X}^{\prime} \mathrm{X}^{\mathrm{e}}$ siècle. Il ressort, par exemple, de la comptabilité de la Société des concerts du Conservatoire pour 1830 que $34 \%$ des dépenses était consacré au payement de ce droit.

Quelques rares concerts étaient demandés par de hautes personnalités de la Cour. Le 11 mars 1830, l'Institution de Choron (subventionnée, il est vrai) donnait un concert «par ordre exprès» et "en présence de la duchesse de Berri». La même demanda, le 30 mai suivant, à la Société des concerts une séance extraordinaire à son intention. Après la révolution de juillet ces interventions disparurent.

Dès 1830 on trouve des traces de l'activité de Guillaume-Louis Bocquillon dit Wilhem, l'initiateur du mouvement orphéonique, alors entièrement dirigé vers l'enseignement du chant choral dans les écoles primaires. La Société pour l'instruction élémentaire organisait des auditions publiques, notamment à l'Hôtel de ville. Le 17 avrii 1830 , pour son assemblée générale annuelle, elle y présenta le concert suivant, dirigé par Wilhem:

«Du ciel éternelle harmonie», chant solennel suivi de couplets sur l'air de Brennus par d'Epagny, musique de Wilhem.

«Invocation à l'amitié», tirée du Paria de C. Delavigne, choeur à trois parties, musique de H. Aymon.

Couplets de distribution des prix par B. Delaroche sur un air allemand, dialogues et arrangements à quatre parties vocales par Wilhem.

Honneur, honneur!, hommage aux propagateurs de l'enseignement élémentaire, choeur à quatre parties avec solos par Wilhem.

Les 80 choristes provenaient de deux écoles de jeunes filles et d'une école protestante de garçons. L'idéologie de la révolution de juillet n'était pas assez «sociale» pour amener le nouveau pouvoir à soutenir le mouvement de Wilhem, qui dut se contenter de l'appui des communes. ${ }^{1}$

1 Voir sur ce sujet le récent article de Paul Gerbod, "L'institution orphéonique en France du XIXe au XXe'siècle», dans Ethnologie française, 1980 , p. $27-44$. 
On peut seulement signaler l'existence marginale d'une activité à caractère folklorique: quatre «chanteurs tyroliens» se font entendre à l'Opéra-comique en janvier 1830 et les salons de Pape accueillent à nouveau, le 16 janvier 1831, ces "chanteurs des Alpes» (Kreipl, Freudenschuss, Schmiảt et Abbiati), qui débutent naturellement par le Ranz des vaches de Leoben. Quant à la musique des innombrables bals publics de toutes catégories - qui tiennent une grande place dans la vie sociale du temps (cf. notamment Balzac) - on ne les envisagera pas ici.

Il nous reste à dire un mot du public, pour autant que l'on puisse le définir. Les seuls concerts dont les listes d'abonnés soient conservées sont ceux de Baillot (Archives Daniel Lainé, Paris), qui paraissent réunir une société particulièrement élitiste, tandis que ceux de la Société des concerts du Conservatoire manquent précisément pour les deux années qui ont fait l'objet de notre enquête.

Mais nous disposons de quelques tentatives de définitions des publics parisiens en 1830. Un journaliste de La Mode, qui n'a pas signé son article («De la musique en France») mais qui est sans doute Miel, compare en février-mars de cette année les différentes façons de réagir devant la musique en Allemagne, en Italie et en France. Reconnaissant la difficulté de son propos, il constate d'abord l'hétérogénéité et les discordances de ce public. Il y a en premier lieu la foule des «stationnaires», c'est-à-dire des réactionnaires, amateurs de vaudevilles, de romances et de contredances, qui, devant les oeuvres de l'école moderne, ne trouvent que «tapage et confusion». Puis les dilettanti, «race superbe dans ses goûts, tranchante dans ses décisions, exclusive surtout et esclave de mille préjugés», trop enfermée dans son snobisme, dans Othello, la Gazza et le Barbier. "C'est pourtant ce même public, continue-t-il, qui court avec tant d'empressement aux concerts graves et savants du Conservatoire, parce que de vrais connaisseurs l'ont averti que c'était chose merveilleuse. C'est lui qui y écoute avec quelque intelligence, qui applaudit avec transport. Il semble qu'il ait laissé dehors ses préventions et son orgueil: il sent et comprend quelquefois».

Le tableau que nous avons tracé de la vie foisonnante des concerts du Paris d'Hernani, du Rouge et le noir et de la Peau de chagrin pourra aider à comprendre la réaction du jeune Frédéric Chopin qui, arrivant de Varsovie, Berlin, Munich et Vienne, a cette réflexion devant le vie musicale de la capitale française en 1831: «A Paris, j'ai tout comme je ne l'ai jamais eu... Le nombre de gens s'intéressant à quelque branche de l'art musical est surprenant». 


\section{POVZETEK}

Avtor želi na osnovi obstoječih virov prikazati koncertno življenje v Parizu v letih 1830 in 1831. Po uvodu, v katerem navaja število koncertov, trajanje koncertne sezone, razporeditev koncertov, višino vstopnin, itd., preide avtor na osrednji del, kjer poizkuša koncerte razporediti na določene tipe. Najprej so tu koncerti raznih glasbenih ustanov in društev, ki pa se med seboj razlikujejo tako po vsebini kot namenu, sledijo koncerti komorne glasbe, potem t. i. koncerti virtuozov, ki so najpogostejši $\mathrm{v}$ teh letih, koncerti $\mathrm{v}$ dobrodelne namene in tisti, katerih izkupiček je bil namenjen glasbenikom $v$ denarnih težavah. Pod Karlom X. je tudi sam kralj kdaj kaj pridal in to je bila edina oblika subvencije oblasti, kajti sicer so bili koncerti močno obdavčeni. Redki so bili koncerti, ki jih je naročil dvor. Od leta 1830 dalje so že vidni sadovi gibanja za zborovsko petje, ki organizira javne nastope in končno, kot obrobna zanimivost, so tu še koncerti folklorne skupine štirih tirolskih pevcev.

Avtor spregovori tudi o koncertni publiki, čeprav je to zelo težko, saj je seznamov abonentov in člankov o reakcijah koncertne publike zelo malo. Iz obstoječega avtor sklepa, da je bilo občinstvo zelo raznoliko in da so bili tudi njegovi interesi zelo različni. Članek se zaključi z ugotovitvijo, da je bilo zanimanje $\mathrm{za}$ glasbo $\mathrm{v}$ Parizu $\mathrm{v}$ teh letih presenetljivo bogato in raznovrstno, kot je ob svojem prihodu v Pariz lahko ugotovil Chopin. 